\title{
PROPAGATION OF WAVES IN THE LAYER OF A THERMO-VISCOELASTIC TRANSVERSELY ISOTROPIC MEDIUM
}

\author{
R.R. GUPTA* \\ Department of CS\&IT, Mazoon College \\ Muscat, Sultanate of Oman, OMAN \\ E-mail: raji.mmec@gmail.com \\ R.R. GUPTA \\ Department of Mathematics and Applied Sciences MEC \\ Muscat, Sultanate of Oman, OMAN \\ E-mail:dr.rajanigupta@gmail.com
}

\begin{abstract}
The article is presented to enhance our knowledge about the propagation of Lamb waves in the layer of a viscoelastic transversely isotropic medium in the context of thermoelasticity with GN theory of type-II and III. Secular equations for symmetric and skew-symmetric modes of wave propagation in completely separate terms are derived. The amplitudes of displacements and temperature distribution were also obtained. Finally, the numerical solution was carried out for cobalt and the dispersion curves, amplitudes of displacements and temperature distribution for symmetric and skew-symmetric wave modes are presented to evince the effect of anisotropy. Some particular cases are also deduced.
\end{abstract}

Key words: viscoelastic, thermoelasticity, phase velocity transversely isotropic.

\section{Introduction}

The propagation of waves in thermoelastic materials has many applications in various fields of science and technology, namely, atomic physics, industrial engineering, thermal power plants, submarine structures, pressure vessel, aerospace, chemical pipes and metallurgy. The importance of thermal stresses in causing structural damages and changes in functioning of the structure is well recognized whenever thermal stress environments are involved. Therefore, the ability to predict electro-dynamics stress induced by a sudden thermal loading in composite structures is essential for the proper and safe design and the knowledge of its response during the service in these severe thermal environments.

The classical theory of thermo-elasticity involving infinite speed of propagation of thermal signals, contradicts the physical facts. During the last three decades, non-classical theories involving finite speed of heat transportation in elastic solids have been developed to remove this paradox. In contrast to the conventional coupled thermo-elasticity theory [1], which involves a parabolic-type heat transport equation, these generalized theories involve a hyperbolic-type heat transport equations, that are supported by experiments exhibiting the actual occurrence of wave-type heat transport in solid, called the second sound effect. The extended thermo-elasticity theories proposed by Lord and Shulman [2], incorporated a flux- rate term into Fourier's law of heat conduction, and formulated a generalized form that involves a hyperbolictype heat transport equation and admits finite speed of thermal signal. Green and Lindsay [3] developed temperature-rate-dependent thermo-elasticity theory by introducing relaxation time factors that does not

\footnotetext{
* To whom correspondence should be addressed
} 
violate the classical Fourier law of heat conduction and this theory also predicts a finite speed for heat propagation.

In recent surveys, Chandrasekharaiah [4], Hetnarski and Ignazack [5] considered the theory proposed by Green and Naghdi [6-9] as an alternate way of formulating the propagation of heat. This theory is developed in a rational way to produce a fully consistent theory that is capable of incorporating thermal pulse transmission in a very logical manner. They make use of general entropy balance rather than an entropy inequality. The development is quite general and the characterization of material response for the thermal phenomena is based on three types of constitutive function that are labeled as type I, type II, and type III. When theory of type I is linearized, the parabolic equation of heat conduction arises. Here, we are interested in the theory of type II (a limiting case of the type III), which does not admit energy dissipation. This theory is usually called "without energy dissipation". Following Green and Naghdi, the theory of thermoelasticity without energy dissipation is a good model to explain the heat conduction in continua.

Quintanilla [10] in 2002, proposed a model of the thermoelastic theory without energy dissipation for materials with affine microstructure. In this article he obtained the equations for the linear theory and also obtained uniqueness theorem for materials with a centre of symmetry. Taheri et al. [11] and Puri et al. [12] employed the Green-Naghdi linear theory of thermoelasticity of types II and III to study the thermal and mechanical waves in a layer of homogeneous thermoelastic solid and plane waves, respectively. Many researchers investigated different types of problems in the theory of thermoelasticity of type III [13-20]. The inelastic behavior of the Earth's material plays an important role in changing the characteristics of seismic waves, in defining seismic source functions Brune [21], and in determining the internal structure of the Earth. The general theory of viscoelasticity describes the linear behavior of both elastic and inelastic materials and provides the basis for describing the attenuation of seismic waves due to inelasticity.

The main objective of the present paper is to study the propagation of Lamb waves in the layer of a viscoelastic transversely isotropic medium in the context of thermoelasticity with GN theory of type-II and III. This study has many applications in various fields of science and technology, namely, atomic physics, industrial engineering, thermal power plants, submarine structures, pressure vessel, aerospace, chemical pipes and metallurgy. After developing the solution, frequency equations connecting the phase velocity with the wave number, for symmetric and skew-symmetric wave modes are derived. The amplitude ratios of displacements and temperature distribution are also obtained. The dispersion curves, attenuation coefficients, amplitude ratio of displacements and temperature distribution for symmetric and skew-symmetric waves are presented and illustrated graphically to evince the effect of anisotropy.

\section{Basic equations}

The constitutive relations and balance laws in a general anisotropic viscoelastic medium in the context of thermoelasticity with GN theory of type-II and III, possessing a center of symmetry, in the absence of body forces are given by

\section{Constitutive relations:}

$$
t_{i j}=c_{i j k l} e_{k l}-\beta_{i j} T
$$

where

$$
e_{j i}=\frac{1}{2}\left(u_{i, j}+u_{j, i}\right), \quad c_{i j k l}=c_{i j k l}^{\prime}+c_{i j k l}^{\prime \prime} \frac{\partial}{\partial t} .
$$

\section{Balance law:}

$$
t_{i j, j}=\rho \ddot{u}_{i} .
$$




\section{Equation of heat conduction:}

$$
K_{i j} \dot{T}_{, i j}+K_{i j}^{*} T_{, i j}=\rho c^{*} \ddot{T}+T_{o} \beta_{i j} \ddot{u}_{i, j}, \quad i, j=1,2,3
$$

where $t_{i j}$ is the stress tensor, $\rho$ is the bulk mass density, $u_{i}$ is the component of displacement vector, $T$ is the temperature change of a material particle, $T_{o}$ is the reference uniform temperature of the body, $K_{i j}^{*}=k_{i}^{*} \delta_{i j}$ ( $i$ not summed) are the characteristic constants of the theory, $K_{i j}=k_{i} \delta_{i j}$ ( $i$ not summed) is the thermal conductivity, $\beta_{i j}=\beta_{i} \delta_{i j}$ ( $i$ not summed) is the thermal elastic coupling tensor, $c^{*}$ is the specific heat at constant strain, $c_{i j k l}, c_{i j k l}^{\prime}, c_{i j k l}^{\prime \prime}$ are characteristic constants of material and the following symmetric properties are satisfied

$$
c_{i j k l}=c_{k l i j}=c_{j i k l}, \quad K_{i j}^{*}=K_{j i}^{*}, \quad K_{i j}=K_{j i}, \quad \beta_{i j}=\beta_{j i} .
$$

\section{Problem formulation}

We have used appropriate transformations, following Slaughter [21], on the set of Eq.(2.1) to derive equations for a viscoelastic transversely isotropic medium and restricted our analysis to the two dimensional problem. In the present investigation, we consider an infinite layer with traction free surfaces at $x_{3}= \pm H$ (layer of thickness $2 \mathrm{H}$ ), which consists of a homogeneous, viscoelastic transversely isotropic thermoelastic material. We take the origin of the coordinate system $\left(x_{1} ; x_{2} ; x_{3}\right)$ on the middle surface of the layer. The $x_{1}-x_{2}$ plane is chosen to coincide with the middle surface and $x_{3}$ axis normal to it along the thickness. For the two-dimensional problem, we assume the components of the displacement vector of the form

$$
\boldsymbol{u}=\left(u_{1} ; 0 ; u_{3}\right)
$$

and assume that the solutions are explicitly independent of $x_{2}$ i.e., $\frac{\partial}{\partial x_{2}} \equiv 0$. Thus the field equations and constitutive relations for such a medium reduce to

$$
\begin{aligned}
& c_{11} \frac{\partial^{2} u_{1}}{\partial x_{1}^{2}}+c_{55} \frac{\partial^{2} u_{1}}{\partial x_{3}^{2}}+\left(c_{13}+c_{55}\right) \frac{\partial^{2} u_{3}}{\partial x_{1} \partial x_{3}}-\beta_{1} \frac{\partial T}{\partial x_{1}}=\rho \frac{\partial^{2} u_{1}}{\partial t^{2}}, \\
& c_{55} \frac{\partial^{2} u_{3}}{\partial x_{1}^{2}}+c_{33} \frac{\partial^{2} u_{3}}{\partial x_{3}^{2}}+\left(c_{13}+c_{55}\right) \frac{\partial^{2} u_{1}}{\partial x_{1} \partial x_{3}}-\beta_{3} \frac{\partial T}{\partial x_{1}}=\rho \frac{\partial^{2} u_{3}}{\partial t^{2}}, \\
& K_{1} \frac{\partial^{3} T}{\partial t \partial x_{1}^{2}}+K_{3} \frac{\partial^{3} T}{\partial t \partial x_{1}^{2}}+K_{1}^{*} \frac{\partial^{3} T}{\partial x_{1}^{3}}+K_{3}^{*} \frac{\partial^{2} T}{\partial x_{3}^{2}}=\rho c^{*} \frac{\partial^{2} T}{\partial t^{2}}+\beta_{1} T_{o} \frac{\partial^{3} u_{1}}{\partial x_{3} t^{2}}+\beta_{3} T_{o} \frac{\partial^{3} u_{3}}{\partial x_{1} t^{2}},
\end{aligned}
$$

and we have used the notations $11 \rightarrow 1,33 \rightarrow 3,13 \rightarrow 5$ for the material constants.

For further considerations, it is convenient to introduce the non-dimensional variables defined by 


$$
x_{i}^{\prime}=\frac{x_{i}}{L}, \quad u_{i}^{\prime}=\frac{u_{i}}{L}, \quad t_{i j}^{\prime}=\frac{t_{i j}}{c_{55}}, \quad t^{\prime}=\frac{t}{t_{o}}, \quad T^{\prime}=\frac{T}{T_{o}}
$$

where $L, t_{o}, T_{o}$ are parameters having dimensions of length, time and temperature $\left({ }^{\circ} \mathrm{C}\right.$ ), respectively.

\section{Boundary condition}

The boundaries of the plate are assumed to be stress free thermally insulated. Therefore, we consider the following types of boundary conditions:

\section{Mechanical Conditions}

The non-dimensional mechanical boundary conditions at $x_{3}= \pm H$ for stress free and rigidly fixed boundaries, respectively, are given by

$$
t_{33}=0, \quad t_{31}=0
$$

where

$$
t_{33}=c_{13} \frac{\partial u_{1}}{\partial x_{1}}+c_{33} \frac{\partial u_{3}}{\partial x_{3}}-\beta_{3} T
$$

\section{Thermal conditions}

The non-dimensional thermal boundary conditions at $x_{3}= \pm H$ are given by

$$
\frac{\partial T}{\partial x_{3}}+h T=0
$$

where $h$ is the surface heat transfer coefficient;

$h \rightarrow 0$ corresponds to thermally insulated boundaries and

$h \rightarrow \infty$ refers to isothermal boundaries.

\section{Normal mode analysis and solution of the problem}

We assume the solution for $u_{1}, u_{3}, T$ representing propagating waves in the $x_{1}-x_{3}$ plane of the form

$$
\left(u_{1}, u_{3}, T\right)=\left(1, \boldsymbol{u}_{3}, \boldsymbol{T}\right) u_{1} e^{i \xi\left(x_{1}+m x_{3}-c t\right)}
$$

where $\xi$ is the wave number, $\omega=\xi_{c}$ is the angular frequency and $c$ is the phase velocity of the wave, $m$ is the unknown parameter which signifies the penetration depth of the wave, $\bar{u}_{3}, \bar{T}$ are, respectively, the amplitude ratios of the displacement $u_{3}$ and temperature distribution $T$ to that of the displacement $u_{1}$.

With the help of Eqs (3.5) and (5.1), Eqs (3.2)-(3.4) reduced to (after suppressing primes) 


$$
\begin{aligned}
& \left(m^{2}+a_{1}+m a_{2} \bar{u}_{3}+a_{3} \bar{T}\right) u_{1} e^{i \xi\left(x_{1}+m x_{3}-c t\right)}=0, \\
& \left(m a_{4}+\left(m^{2}+a_{5}\right) \bar{u}_{3}+a_{6} m \bar{T}\right) u_{1} e^{i \xi\left(x_{1}+m x_{3}-c t\right)}=0, \\
& \left(a_{7}+a_{8} m \bar{u}_{3}+\left(a_{9}-m^{2}\right) \bar{T}\right) u_{1} e^{i \xi\left(x_{1}+m x_{3}-c t\right)}=0
\end{aligned}
$$

where

$$
\begin{aligned}
& a_{1}=\frac{c_{11}}{c_{55}}-\frac{\rho c^{2} L^{2}}{c_{55} t_{o}^{2}}, \quad a_{2}=1+\frac{c_{13}}{c_{55}}, \quad a_{3}=-\frac{1 \beta_{1} T_{o}}{c_{55} \xi}, \\
& a_{4}=\frac{\left(c_{13}+c_{55}\right)}{c_{33}}, \quad a_{5}=\frac{c_{55}}{c_{33}}-\frac{\rho c^{2} L^{2}}{c_{33} t_{o}^{2}}, \quad a_{6}=-1 \frac{\beta_{3} T_{o}}{c_{33} \xi}, \\
& a_{7}=-\frac{1 \beta_{1} L^{2} \xi c^{2}}{t_{o}\left(K_{3} \xi_{c}-K_{3}^{*} t_{o}\right)}, \quad a_{8}=-\frac{1 \beta_{3} L^{2} \xi c^{2}}{t_{o}\left(K_{3} \xi c-K_{3}^{*} t_{o}\right)}, \quad a_{9}=\frac{K_{1}^{*} t_{o}^{2} L-c^{*} \rho L^{3} c^{2}-K_{1} \xi c t_{o} L}{t_{o} L\left(K_{3} 1 \xi c-K_{3}^{*} t_{o}\right)} .
\end{aligned}
$$

The condition for the non trivial solution of system of Eqs (5.2), yields a cubic equation in $m^{2}$ as

$$
m^{6}+A m^{4}+B m^{2}+C=0
$$

where

$$
\begin{aligned}
& A=a_{1}-a_{9}+a_{5}+a_{6} a_{8}-a_{2} a_{4}, \quad C=a_{5}\left(a_{3} a_{7}-a_{1} a_{9}\right), \\
& B=a_{1}\left(a_{5}+a_{6} a_{8}-a_{9}\right)-a_{5} a_{9}+a_{2}\left(a_{4} a_{9}-a_{6} a_{7}\right)+a_{3} a_{7}-a_{3} a_{4} a_{8} .
\end{aligned}
$$

Lamb waves exhibit velocity dispersion, i.e., velocity of propagation $c$ depends on the frequency as well as on elastic constants and density of the materials. These waves are dispersive plate waves that occur for traction-free forces on both surfaces of the plate. The roots of this equation give three values of $\mathrm{m}^{2}$, and hence of $c^{2}$. Three positive values of $c$ will be the velocities of propagation of three possible waves, viz. the quasi-longitudinal wave, quasi-transverse wave and quasi thermal wave. So Eq.(5.3) leads to the following solution for displacements and temperature distribution

$$
\left(u_{1}, u_{3}, T\right)=\sum_{k=1}^{3}\left[A_{k} \cos \left(m_{k} x_{3} \xi\right)+B_{k} \sin \left(m_{k} x_{3} \xi\right)\right]\left(1, r_{k}, t_{k}\right) e^{i \xi\left(x_{1}-c t\right)}
$$

where

$$
r_{k}=-\frac{m_{k}\left[a_{4} m_{k}^{2}-\left(a_{4} a_{9}-a_{6} a_{7}\right)\right]}{m_{k}^{4}-m_{k}^{2}\left(a_{9}-a_{5}-a_{6} a_{8}\right)-a_{5} a_{9}}, \quad t_{k}=-\frac{\left(a_{4} a_{8}-a_{7}\right) m_{k}^{2}-a_{5} a_{7}}{m_{k}^{4}-m_{k}^{2}\left(a_{9}-a_{5}-a_{6} a_{8}\right)-a_{5} a_{9}} .
$$




\section{Derivation of secular equation}

Substituting the values of $u_{1}, u_{2}$ and $T$ in the boundary conditions given by Eqs (4.1) and (4.3) at the surfaces $\pm H$ of the layer, together with the help of Eq.(4.2), we obtain

$$
\begin{aligned}
& \sum_{k=1}^{3}\left[\left(\left(g_{1}+g_{2 k}\right) c_{k}-g_{3 i} s_{k}\right) A_{k}+\left(\left(g_{1}+g_{2 k}\right) s_{k}+g_{3 k} c_{k}\right) B_{k}\right]=0, \\
& \sum_{k=1}^{3}\left[-A_{k} g_{6} s_{k}+B_{k} g_{6 k} c_{k}\right]=0, \\
& \sum_{k=1}^{3}\left[\left(\left(g_{1}+g_{2 k}\right) c_{k}-g_{3 k} s_{k}\right) A_{k}+\left(\left(g_{1}+g_{2 k}\right) s_{k}+g_{3 k} c_{k}\right) B_{k}\right]=0, \\
& \sum_{k=1}^{3}\left[-A_{k} g_{6} s_{k}+B_{k} g_{6 k} c_{k}\right]=0, \\
& \sum_{k=1}^{3}\left[\left(g_{4 k} c_{k}-g_{5 k} s_{k}\right) A_{k}+\left(g_{4 k} s_{k}+g_{5 k} c_{k}\right) B_{k}\right]=0, \\
& \sum_{k=1}^{3}\left[\left(g_{4 k} c_{k}+g_{5 k} s_{k}\right) A_{k}+\left(-g_{4 k} s_{k}+g_{5 k} c_{k}\right) B_{k}\right]=0,
\end{aligned}
$$

where

$$
\begin{aligned}
& s_{k}=\sin \left(m_{k} \xi x_{3}\right), \quad c_{k}=\cos \left(m_{k} \xi x_{3}\right), \quad g_{1}=\frac{c_{13}}{c_{33}} \xi, \quad g_{2 k}=\frac{\beta_{3} t_{k}}{c_{13}}, \\
& g_{3 k}=r_{k} m_{k} \xi, \quad g_{4 k}=\frac{c_{55}}{c_{33}} 1 r_{k} \xi, \quad g_{5 k}=\frac{c_{55}}{c_{33}} m_{k} \xi, \quad g_{6 k}=t_{k} m_{k} \xi, \quad k=1,2,3 .
\end{aligned}
$$

In order to satisfy the six boundary conditions given by Eqs (4.1) and (4.3) simultaneously, the determinant of the coefficients of $A_{k}$ and $B_{k}, k=1,2,3$ in Eqs (6.1) must vanish. This gives an equation for the frequency of the layer oscillations. The frequency equation for the waves in the present case, after applying lengthy algebraic reductions and manipulations of the determinant leads to the following secular equation

$$
\begin{aligned}
& {\left[T_{1}\right]^{ \pm}\left[g_{61} g_{42}\left(g_{1}+g_{23}\right)-g_{61} g_{43}\left(g_{1}+g_{22}\right)\right]+} \\
& +\left[T_{2}\right]^{ \pm}\left[g_{62} g_{43}\left(g_{1}+g_{21}\right)-g_{62} g_{41}\left(g_{1}+g_{23}\right)\right]+ \\
& +\left[T_{3}\right]^{ \pm}\left[g_{63} g_{41}\left(g_{1}+g_{22}\right)-g_{63} g_{42}\left(g_{1}+g_{21}\right)\right]=0 .
\end{aligned}
$$

These are the frequency equations which correspond to the symmetric and skew symmetric modes with respect to the medial plane $x_{3}=0$. Here,

$$
T_{k}=\tan \left(m_{k} \xi x_{3}\right), \quad k=1,2,3 .
$$


The solution of this equations also reveals the precise form of the particle motion, which Eq.(5.4) represents in a generic form only. It is found that in Eq.(6.2) the superscript ' + ' gives rise to a family of waves whose motion is symmetrical about the mid-plane of the plate (the plane $x_{3}=0$ ), while the superscript ' - ' gives rise to a family of waves whose motion is anti-symmetrical about the mid-plane.

\subsection{Amplitude of displacements and temperature distribution}

In this section, the amplitude of displacement components and temperature distribution for symmetric and skew symmetric mode of plane waves can be obtained as

$$
\begin{aligned}
& \left(\left(u_{1}\right)_{s y},\left(u_{1}\right)_{a s y}\right)=\sum_{k=1}^{3}\left(A_{k} \cos \left(\xi m_{k} x_{3}\right), B_{k} \sin \left(\xi m_{k} x_{3}\right)\right) e^{i \xi\left(x_{1}-c t\right)}, \\
& \left(\left(u_{2}\right)_{s y},\left(u_{2}\right)_{a s y}\right)=\sum_{k=1}^{3} r_{k}\left(A_{k} \sin \left(\xi m_{k} x_{3}\right), B_{k} \cos \left(\xi m_{k} x_{3}\right)\right) e^{1 \xi\left(x_{1}-c t\right)}, \\
& \left((T)_{s y},(T)_{a s y}\right)=\sum_{k=1}^{3} t_{k}\left(A_{k} \cos \left(\xi m_{k} x_{3}\right), B_{k} \sin \left(\xi m_{k} x_{3}\right)\right) e^{i \xi\left(x_{1}-c t\right)}
\end{aligned}
$$

\subsection{Specific loss}

The specific loss is the ratio of the energy dissipated $(\Delta W)$ in taking a specimen through a stress cycle to the elastic energy $W$ stored in the specimen when the strain is a maximum. For a plane sinusoidal wave of small amplitude, Kolsky [22] shows that the specific loss $\frac{\Delta W}{W}$ is $4 \pi$ times the absolute value of the ratio of the imaginary part to the real part of, i.e.

$$
\frac{\Delta W}{W}=4 \pi\left|\frac{\operatorname{Im}(\xi)}{\operatorname{Re}(\xi)}\right|
$$

He noted that the specific loss is the most direct method of defining internal friction for a material.

\section{Numerical results and discussion}

In order to illustrate the theoretical results obtained in the preceding sections, we now present some numerical results. The following relevant physical constants for cobalt material are taken from Dhaliwal and Singh [23] for a thermoelastic transversely isotopic material,

$$
\begin{array}{lll}
c_{11}^{\prime}=3.071 \times 10^{11} \mathrm{~N} / \mathrm{m}^{2}, & c_{12}^{\prime}=1.650 \times 10^{11} \mathrm{~N} / \mathrm{m}^{2}, & c_{13}^{\prime}=1.027 \times 10^{11} \mathrm{~N} / \mathrm{m}^{2}, \\
c_{33}^{\prime}=3.581 \times 10^{11} \mathrm{~N} / \mathrm{m}^{2}, & c_{5511}^{\prime}=1.51 \times 10^{11} \mathrm{~N} / \mathrm{m}^{2}, & \beta_{1}=7.04 \times 10^{6} \mathrm{~N} / \mathrm{m}^{2} \mathrm{~K}, \\
\beta_{3}=6.90 \times 10^{6} \mathrm{~N} / \mathrm{m}^{2} \mathrm{~K}, & \rho=8.836 \times 10^{3} \mathrm{Kg} / \mathrm{m}^{3}, & K_{1}=6.90 \times 10^{2} \mathrm{~W} / \mathrm{m} . \mathrm{K}, \\
K_{3}=7.01 \times 10^{2} \mathrm{~W} / \mathrm{m} . \mathrm{K}, & K_{1}^{*}=1.313 \times 10^{2} \mathrm{~W} . \mathrm{sec}, & K_{3}^{*}=1.54 \times 10^{2} \mathrm{~W} . \mathrm{sec}, \\
c^{*}=4.27 \times 10^{2} \mathrm{~J} / \mathrm{Kg} . \mathrm{K}, & T=298 \mathrm{~K} .
\end{array}
$$


For a particular model of a thermo-visco-elastic transversely isotropic solid the relevant parameters are expressed as

$$
\begin{aligned}
& c_{11}=c_{11}^{\prime}\left(1+\mathrm{\imath} Q_{1}^{-1}\right), \quad c_{12}=c_{12}^{\prime}\left(1+\mathrm{\imath} Q_{2}^{-1}\right), \quad c_{13}=c_{13}^{\prime}\left(1+\mathrm{\imath} Q_{3}^{-1}\right), \\
& c_{33}=c_{33}^{\prime}\left(1+\mathrm{\imath} Q_{4}^{-1}\right), \quad c_{55}=c_{55}^{\prime}\left(1+\mathrm{\imath} Q_{5}^{-1}\right)
\end{aligned}
$$

where

$$
Q_{1}^{-1}=10.05, \quad Q_{2}^{-1}=3.04, \quad Q_{3}^{-1}=3.05, \quad Q_{4}^{-1}=.005, \quad Q_{5}^{-1}=2.05
$$

The corresponding plots of non-dimensional phase velocity, attenuation coefficient and the specific loss with non-dimensional real part of wave number $(R)$ in the case of $H=l$ for symmetric and skew-symmetric modes are given in Figs 1-6. Here, a solid line with and without a center symbol represents the variations corresponding to the variations for a viscoelastic transversely isotropic medium in the context of thermoelasticity with GN theory of type-II and III (TIVWED) and, for comparison, broken lines with and without a center symbol represent the variations corresponding to a transversely isotropic thermoelastic solid (TIWED). The lines shown in the figures without a center symbol represent the variations corresponding to the initial mode $(n=1)$ of wave propagation, lines with a center symbol $(o)$ represent the variations corresponding to the second mode $(n=2)$ and lines with a center symbol $(x)$ represent the variations corresponding to the final mode $(n=3)$ of wave propagation.

Figures 1 and 4 show the variations of phase velocity with respect to wave number for symmetric and skew symmetric modes, respectively. It is clear from Fig. 1 that the value of phase velocity for all the modes of wave propagation, sharply decreases and flattens out to become constant at the end for both the cases of (TIVWED) and (TIWED). However, the variation pattern for the case of the skew symmetric mode is similar to the symmetric mode except its amplitude of oscillation.

Figures 2 and 5 show the variation of the attenuation coefficient with respect to wave number, for symmetric and skew symmetric modes. The value of attenuation goes on increasing with an increase in wave number for all the modes. It can be seen that the value of the attenuation coefficient for all the modes $(n=1$, $2,3)$ and for both symmetric and skew symmetric modes is lower for TIWED as compared to those of TIVWED.

Figure 3 shows the value of the specific loss for the symmetric mode. It is observed form this figure that for the symmetric mode, the value of specific loss initially appears to be same, and then become higher for the case of TIVWED as compared to those for TIWED, while the reverse behavior is observed for higher modes $(n=2,3)$. While from Fig. 6 it can be seen that the value of the specific loss for all the modes increases with an increase in wave number and the values for TIVWED is higher as compared to those of TIWED.

Figures 5-7 show the variations of amplitude ratios of $u_{1}, u_{3}$ and $T$ with respect to the thickness of layer for symmetric and skew symmetric modes. It is observed from Fig.5 that the amplitude ratio of $u_{1}$ alternately increases or decreases with an increase in the thickness of layer for the skew symmetric mode and oscillates with a very small amplitude for the symmetric mode in the case of (TIVWED). However for (TIWED), its value initially decreases and then slowly increases with an increase in $H$. The magnitude of the amplitude ratio of $u_{l}$ is decreased due to anisotropy. It is observed from Fig. 6 that the value of the amplitude ratio of normal displacement $u_{3}$ for the skew symmetric mode is initially constant, then decreases sharply and then increases with an increase in $H$ and for the symmetric mode it increases with an increase in $H$ for (TIVWED). However, for (TIWED) its value increases with an increase in $x$ for both the symmetric and skew symmetric modes. It is evident from Fig.7 that the variations in the value of the amplitude ratio of $T$ is similar to that of the normal displacement $u_{3}$ for (TIVWED), while for (TIWED) and both the symmetric and skew symmetric mode its value initially increases then remains constant and with a further increase in $H$ its value decreases with thickness $H$. 


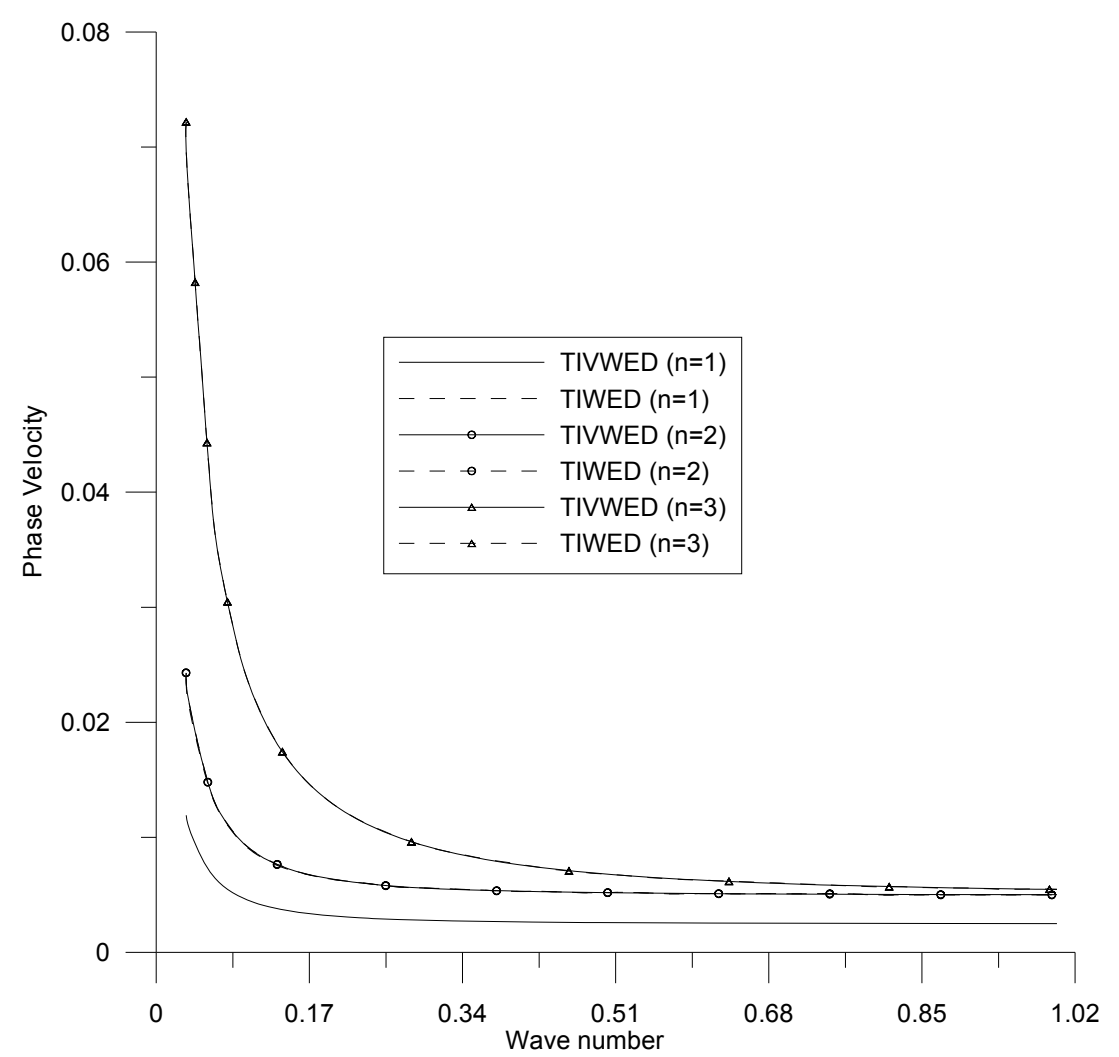

Fig.1. Variations of phase velocity with wave number for symmetric mode.

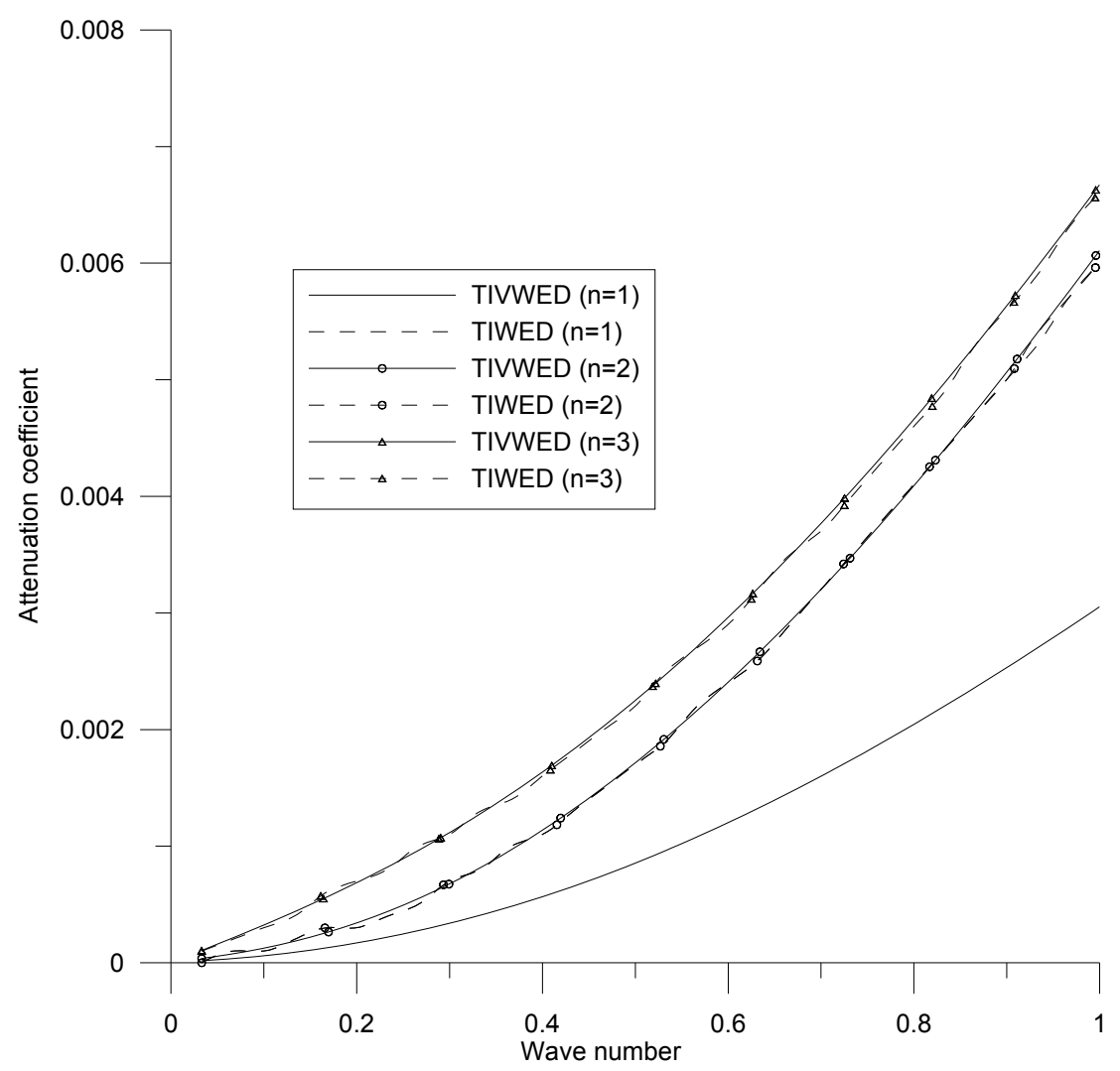

Fig.2. Variations of attenuation coefficient with wave number for symmetric mode. 


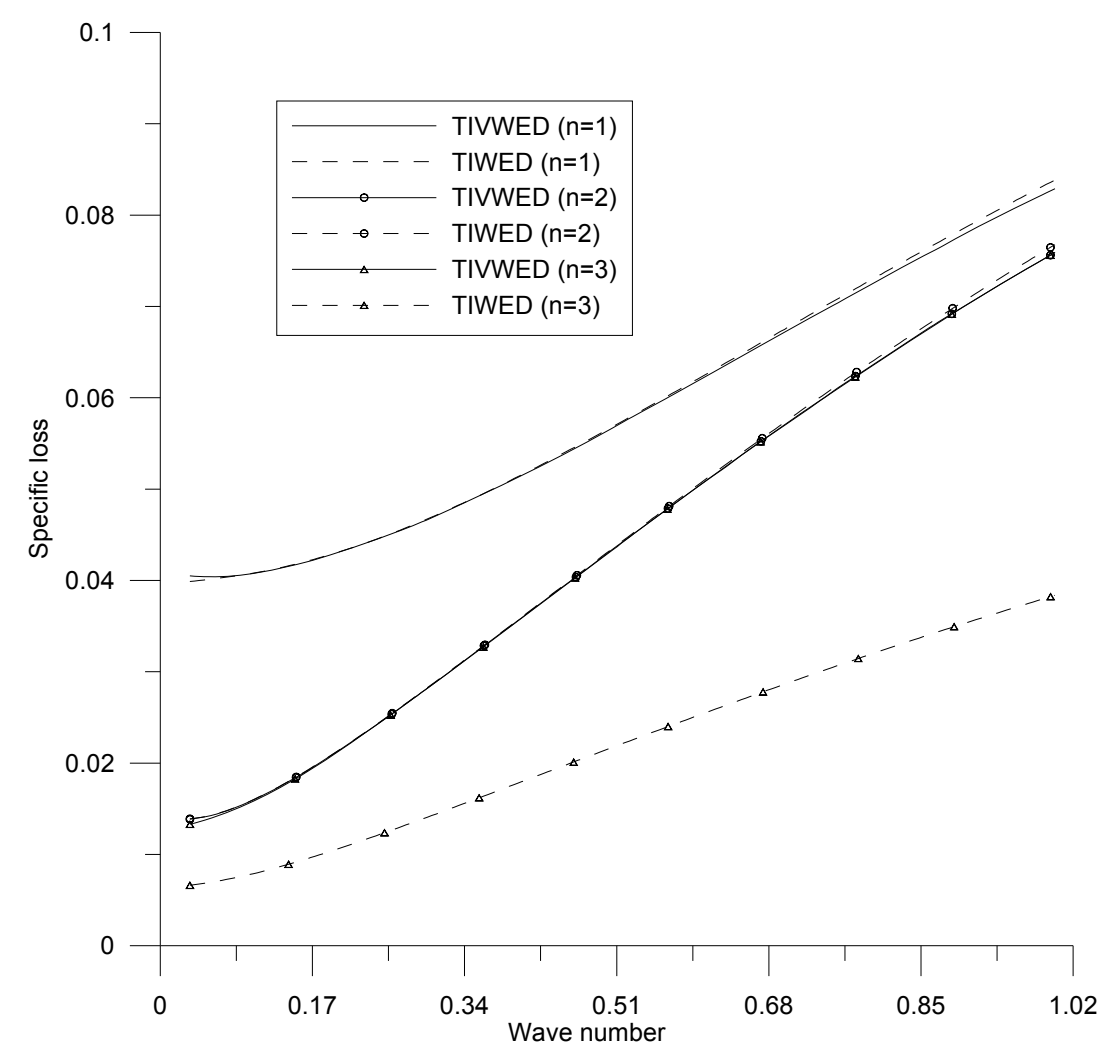

Fig.3. Variations of specific loss with wave number for symmetric mode.

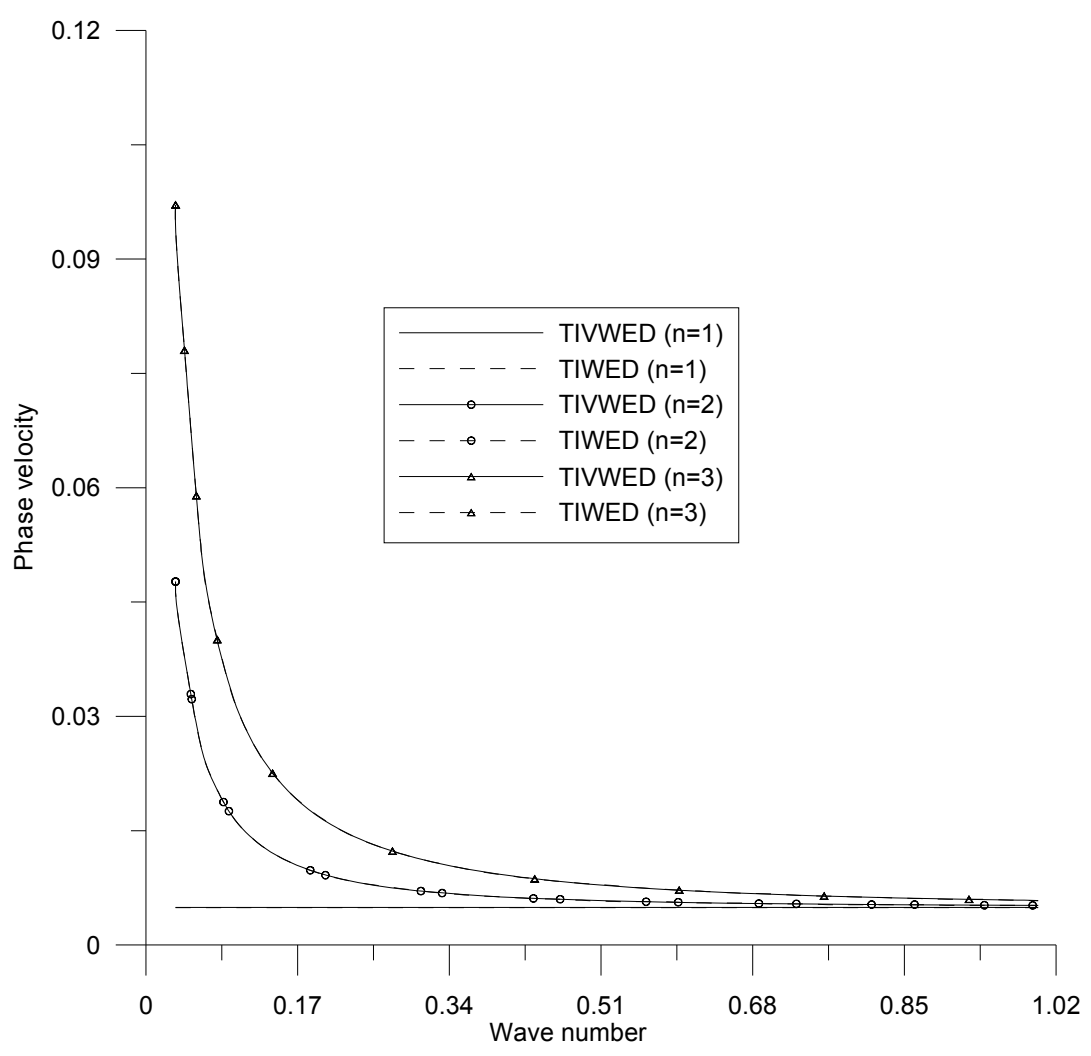

Fig.4. Variations of phase velocity with wave number for skew symmetric mode. 


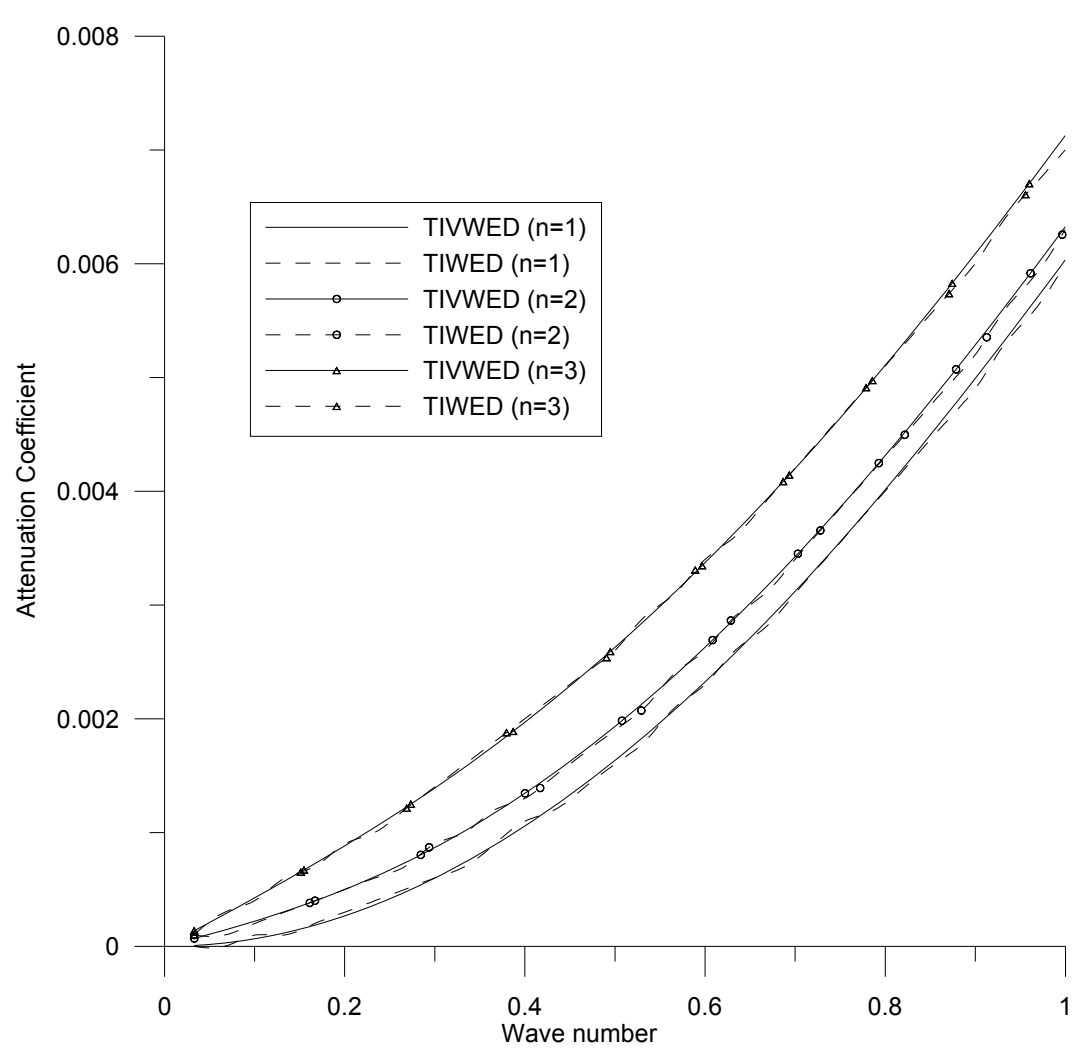

Fig.5. Variations of attenuation coefficient with wave number for skew symmetric mode.

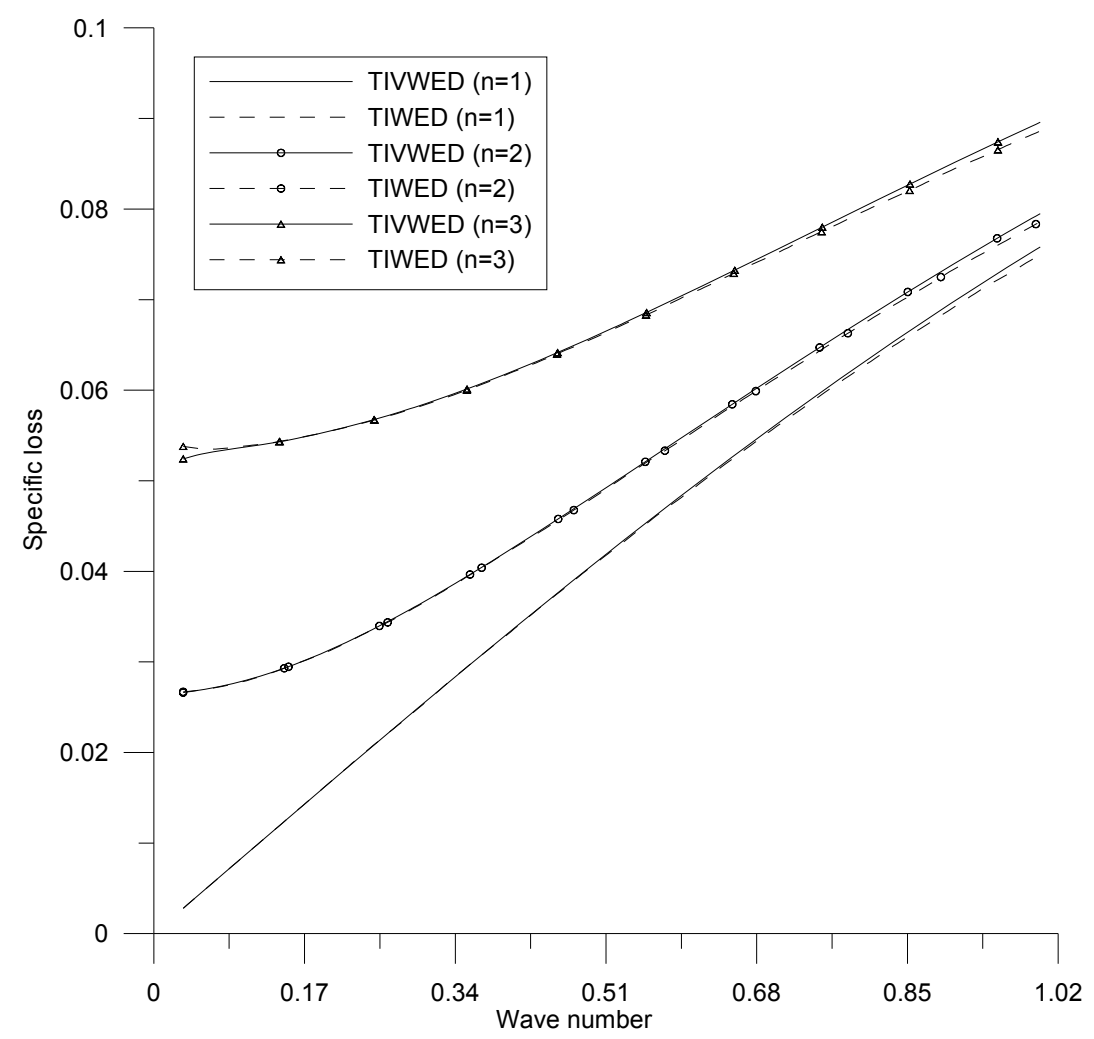

Fig.6. Variations of specific loss with wave number for skew symmetric mode. 


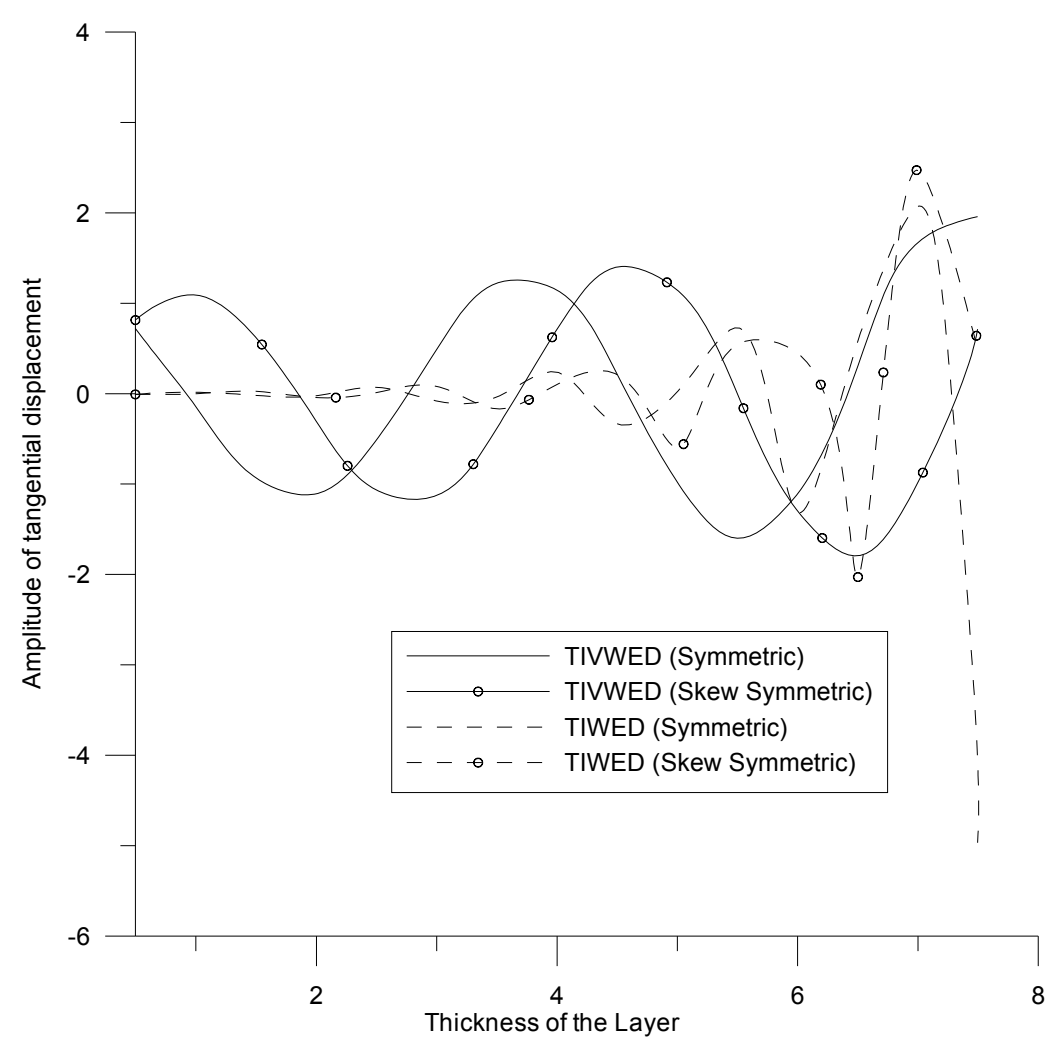

Fig.7. Variations of amplitude of tangential displacement with thickness of the layer.

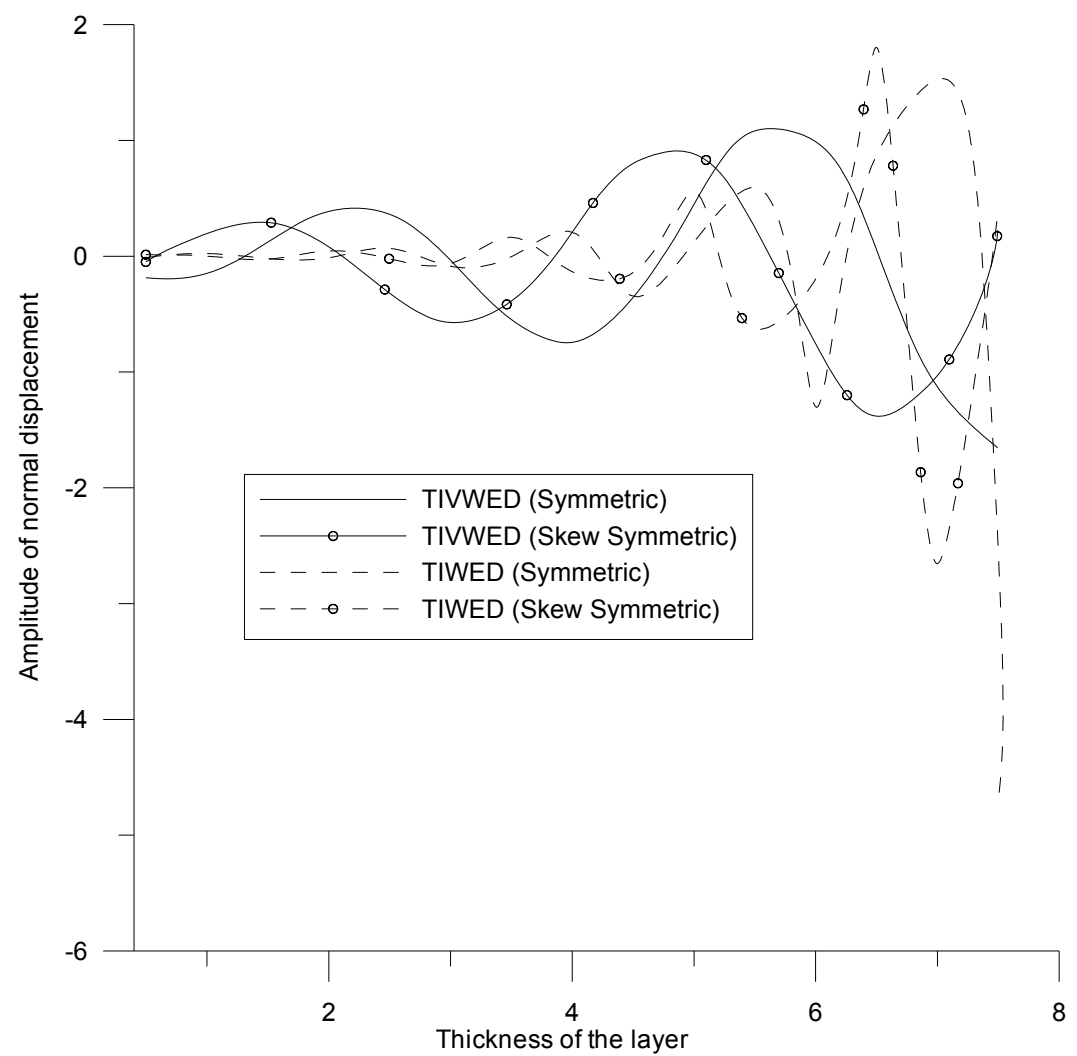

Fig.8. Variations of amplitude of normal displacement with thickness of the layer. 


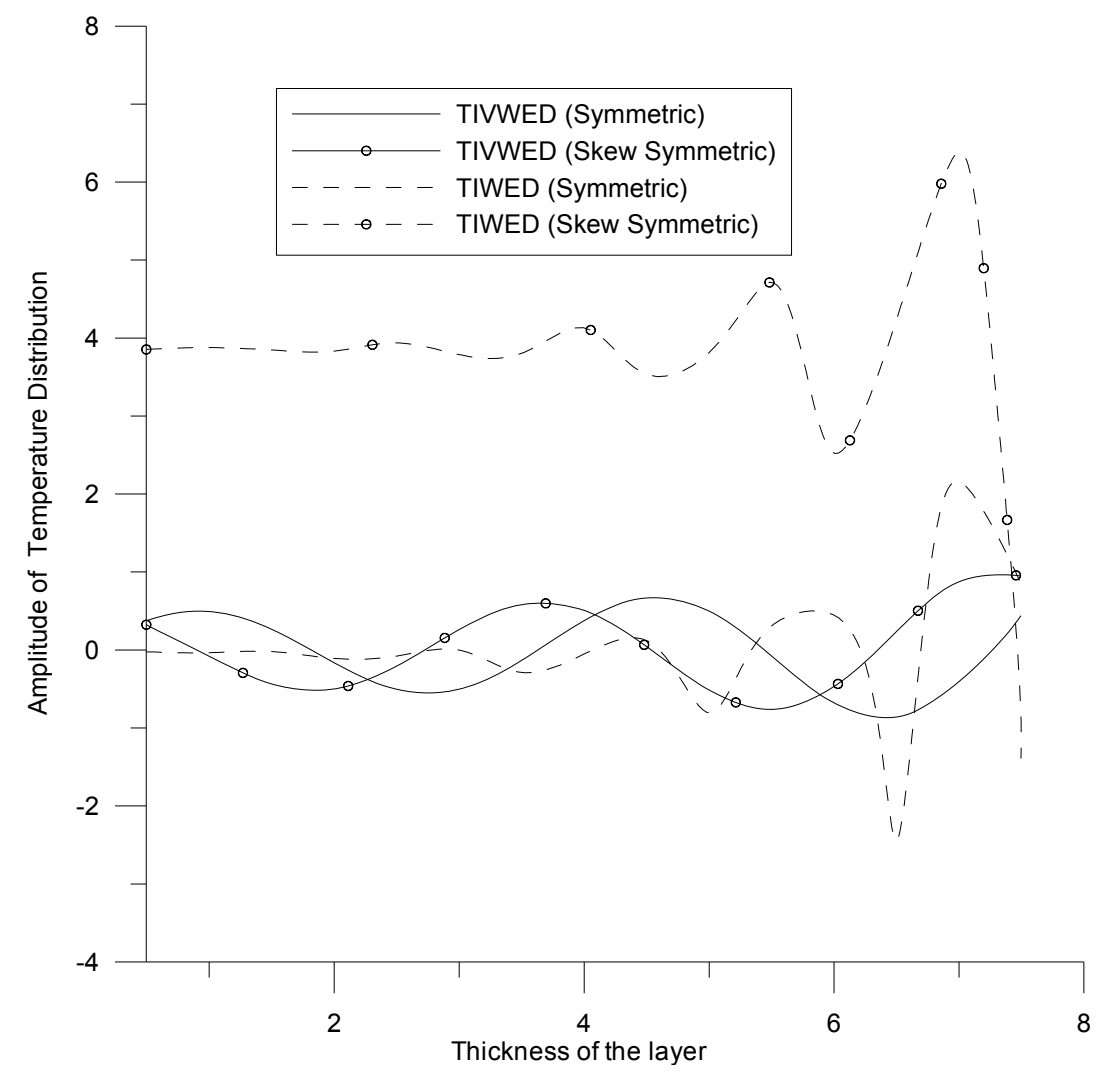

Fig.9. Variations of amplitude of temperature distribution with thickness of the layer.

\section{Conclusion}

The propagation of Rayleigh-Lamb waves in an infinite layer of a viscoelastic transversely isotropic medium in the context of thermoelasticity with GN theory of type-II and III deriving the secular equation, is investigate. The waves can propagate long distances and are used for damage detection of plate-like structures. They are extremely useful for detection of cracks in thin sheet materials and tubular products, since these waves will travel several meters in steel and so are useful to scan plates, wires and tubes. Lamb waves are the most widely used guided waves for damage detection.

\section{Nomenclature}

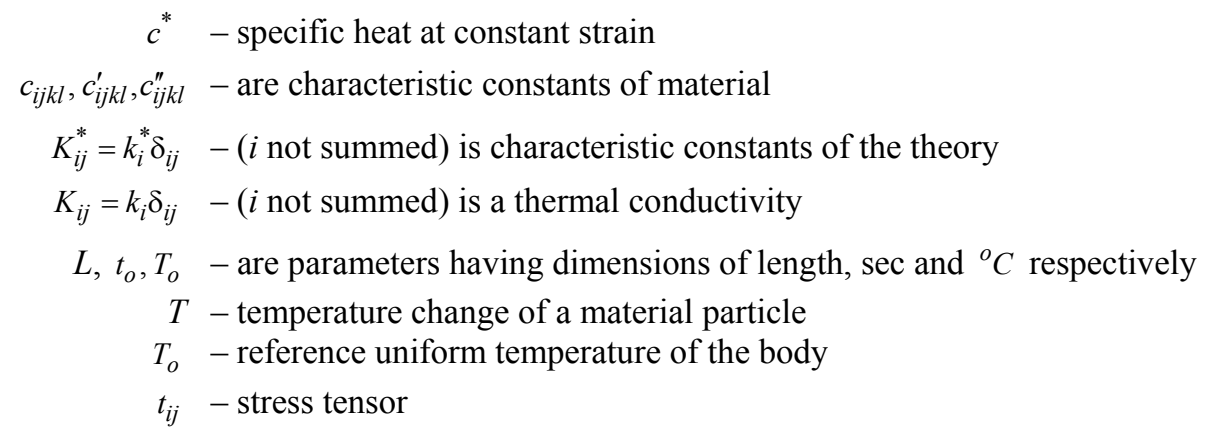


$u_{i}$ - component of displacement vector

$\beta_{i j}=\beta_{i} \delta_{i j}-(i$ not summed $)$ the thermal elastic coupling tensor

$\rho \quad$ - bulk mass density

The comma notation is used for spatial derivatives and superimposed dot represents time differentiation.

\section{References}

[1] Brune J.N. (1970): Tectonic stress and the spectra of seismic shear waves from earthquakes. - [J]. J. Geophys. Res., vol.75, pp.4997-5009.

[2] Lord H. and Shulman Y.A. (1967): Generalized dynamical theory of thermoelasticity. - J. Mech. Phys. Solid, vol.15, pp.299-309.

[3] Green A.E. and Lindsay K.A. (1972): Thermoelasticity. - J. Elasticity, vol.2, pp.1-5.

[4] Chandrasekhariah D.S. (1998): Hyperbolic thermoelasticity: A review of recent literature. - Appl. Mech. Rev., vol.51, pp.705-729.

[5] Hetnarski R.B. and Iganaczak J. (1999): Generalized thermoelasticity. - J. Thermal Stresses, vol.22, pp.451-470.

[6] Green A.E. and Naghdi P.M. (1995): A united procedure for construction of theories of deformable media. I. Classical continuum physics, II. Generalized continua, III. Mixtures of Interacting continua. - Proc. Royal Soc. London Ser. A, vol.448, pp.335-356, pp.357-377, pp.379-388.

[7] Green A.E. and Naghdi P.M. (1991): A re-examination of the basic postulates of thermomechanics. - Proc. Royal Soc. London Ser. A, vol.432, pp.171-194.

[8] Green A.E. and Naghdi P.M. (1992): On undamped heat waves in an elastic solid. - J. Thermal Stresses, vol.15, pp.253-264.

[9] Green A.E. and Naghdi P.M. (1993): Thermoelasticity without energy dissipation. - J. Elasticity, vol.31, pp.189208.

[10] Quintanilla R. (2002): Thermoelasticity without energy dissipation of materials with microstructure. - Applied Mathematical Modelling, vol.26, pp.1125-1137.

[11] Taheri H., Fariboz S. and Eslami M.R. (2004): Thermoelasticity solution of a layer using the Green-Neghdi model. - Journal of Thermal Stresses, vol.27, pp.795-809.

[12] Puri P. and Jordan P.M. (2004): On the propagation of plane waves in type-III thermoelastic media. - Proc. R. Soc. London. A, vol.460, pp.3203-3221.

[13] Lazzari B. and Nibbi R. (2008): On the exponential decay in thermoelasticity without energy dissipation an of type III in prence of an absorbing boundary. - J. Math. Anal. Appl., vol.338, pp.317-329.

[14] Roychoudhuri S.K. and Bandyopadhyay N. (2007): Interactions due to body forces in generalized thermo-elasticity III. - Comptures and Mathematics with Applications, vol.54, pp.1341-1352.

[15] Mukhopadhyay S. and Roushan Kumar (2008): A problem on thermoelastic interactionsin an infinite medium with a cylindrical hole in generalized thermoelasticity III. - Journal of Thermal Stresses, vol.31, pp.455-457.

[16] Quintanilla R. and Racke R. (2003): Stability inthermoelasticity of type III. - Discrete and Continuous Dynamical Systems-Series B, vol.3, No.3, pp.383-400.

[17] Quintanilla R. (2009): Type II thermoelasticity. A new aspect. - Journal of Thermal Stresses, vol.32, pp.290-307.

[18] Quintanilla R. (2001): Structural stability and continuous dependence of solutions of thermoelasticity of type III. Discrete and Continuous Dynamical Systems-Series B, vol.1, No.4, pp.463-470. 
[19] Quintanilla R. and Straughan B. (2004): A note on discontinuity waves in type III thermoelasticity. - Proc. R. Soc. Lond. A, vol.460, pp.1169-1175.

[20] Leseduarte M.C. and Quintanilla R. (2006): Thermal stresses in type III thermoelasticplates. - Journal of Thermal Stresses, vol.29, pp.485-503.

[21] Slaughter W.S. (2002): The Linearized Theory of Elasticity. - Birkhauser.

[22] Kolsky H. (1963): Stress Waves in Solids. - Oxford: Clarendon Press; New York: Dover Press.

[23] Dhaliwal R.S. and Singh A. (1980): Dynamic coupled thermoelasticity. - Hindustan Publication Corporation, New Delhi, India, 726.

Received: June 30, 2014

Revised: November 10, 2015 